\title{
Apply Service Oriented Architecture and Web 2.0 Application in Web Services
}

\author{
Ming-Chang Huang \\ Department of Business Information Systems \& Operation Management, University of North Carolina at Charlotte, Charlotte, NC
}

\begin{abstract}
It is important how wireless hosts find other hosts efficiently for load and web service purposes because hosts in an ad-hoc network moves dynamically. I design a three-tier architecture structure for web and load services in ad-hoc computer networks, which is a new system architecture using SOA (service oriented architecture) and Web 2.0 concepts to implement functions for web and load services. This three-tier system structure includes content network, social network and service network, which is based on Web service functions to implement services seeking and load distribution. This paper also constructs a knowledge sharing and learning platform based on this structure. Different communities can provide their services to each other using this new knowledge platform, and it forms a "virtual community." This leads to the desired accomplishments of "service reusability" and "service innovation”. In addition, it also proposes a new framework of SOA, which evolves in other applications in web 2.0 style.
\end{abstract}

Keywords: Web services, Web 2.0, service oriented architecture, load services.

\section{Introduction}

Computer networks can provide parallel computation and services. It is important that hosts find services form other hosts, and send loads to other hosts for certain function implementation through network transfer. With the increasing popularity of mobile communications and computing, the demand for web and load services grows significantly. When a computer is overloaded or it needs special services from other computers, it sends requests to other computers for web and load services. For example, a computer may send requests to other computers when it receives certain jobs that require a higher quality of service or a shorter time that its processor is able to provide. Since wireless networks have been widely used in recent years, how a host finds services it needs or how it transfers loads to other nodes becomes a critically important issue because not all wireless hosts have the ability to manipulate their loads. For

Corresponding author: JongHun Jung, assistant professor, research fields: web and network application programming design, computer networking and communication, data science and analysis, parallel and distributed systems. E-mail: mhuang5@uncc.edu. instance, a host with low battery power cannot finish all jobs on time and should ask other nodes to provide services to complete the jobs, or it should transfer some of jobs to other hosts.

Before a wireless host transfers its loads to other hosts or asks for load services from other hosts, it needs to find all available hosts using resource allocation algorithms. Several resource allocation protocols have been developed, for example, IEFT SLP (service location protocol) [1] and Jini [2] software package from Microsystems. However, these protocols address how to find the resources in wired networks, but not in wireless networks. Maab [3] develops a location information server for location-aware applications based on the X.500 directory service and the lightweight directory access protocol LDAP [4]. However, this approach does not cover several aspects about the movements of mobile hosts, for example, how to generate a new directory service and how a host gets such new service, when a directory agent moves away from its original region. In an Ad-Hoc network, system structure is dynamic and hosts can join or leave any time. Therefore, how 
to provide load services and find available hosts providing load services issues worth exploring in an Ad-Hoc network system. The goal of this paper is explore and confirm that users can easily find and share resources based on the concepts of "service reusability" and "service innovation".

Due to the popularity of Web Services techniques, I build a new architecture in which I adopt the SOA model with Web $2.0[1,5]$ for web services. By using Web 2.0 with SOA, the network resources can be easily found by the hosts which need services. Based on XML, the SOA load service system can be used in any computer system platform [6-8]. This is a very important characteristic for hosts to share or request services across different systems. With the help of Web 2.0, hosts can find the required services easily from the Internet [9].

Fig. 1 shows the basic SOA structure [10-12], which is built by three major components-the Directory, Service consumer, and Service provider. SQA is operated by the following: The Directory provides a platform for information that a service provider can register in the Directory for providing services; a service consumer can find its desired service it needs in the Directory. Once the Directory finds services that a service consumer needs, it sends a query response back to the service consumer to notify it the result. At this time, the service consumer has the information about the hosts which can provide services;

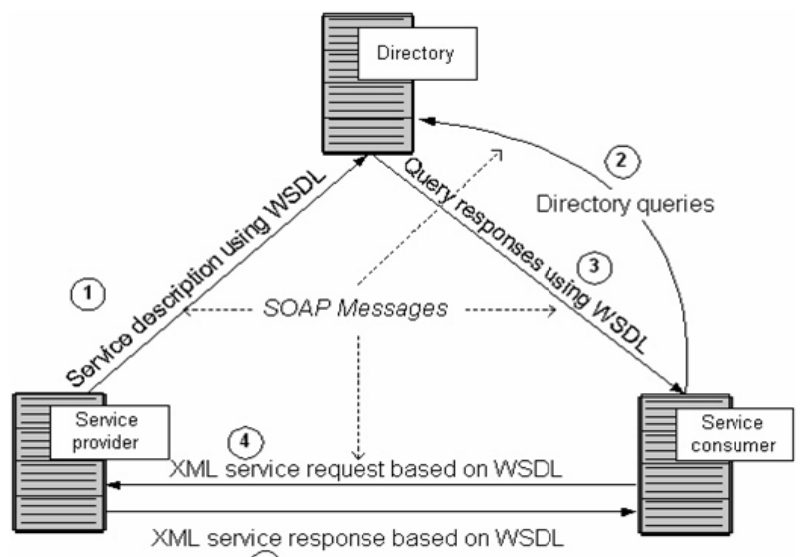

(5)

Fig. 1 SOA structure. therefore the consumer contacts the service provider directory by sending requests. The service provider now will send responses back to the consumer for the services the consumer needs. This is also called the "invoke” process.

\section{System Structure}

The system structure for the SOA model is illustrated in Fig. 2. There are two layers in this structure-the Service Network layer and the Service Logic layer.

The Service Network layer is the main network that connects to the internet using the regular network protocols. It receives requests from internet and forwards requests to the Service Logic layer in WS (Web service) object forms. Each WS object is based on the SOA model which can communicate each other using social network. The Service Logic layer is the main layer that uses WS objects to communicate with each other in the sub-network under the Service Network layer. Different WS object has different objectives, for example, some WS objects are used for social network communication, while other WS objects are used for accessing contents in Content Networks. Since they are in SOA form, it is easy for them to find the resources they need for different purposes. Inside the Service Logic layer, there are sub-networks for different purposes and functions. For example, nodes can form social networks; storage devices can form a content network for data accessing. All these operations are managed by the WS objects under the SOA model. For the service reusability purpose, most WS objects are generated by the Service Network layer for data and object consistency.

In Fig. 2, all the services and requests are in the forms of Web Service objects which are defined and implemented by XML. For users who need services, requests are sent by the users to the Service Network Layer. The Service Network is the gateway for accepting requests and sending back requesting results to the requesters. 


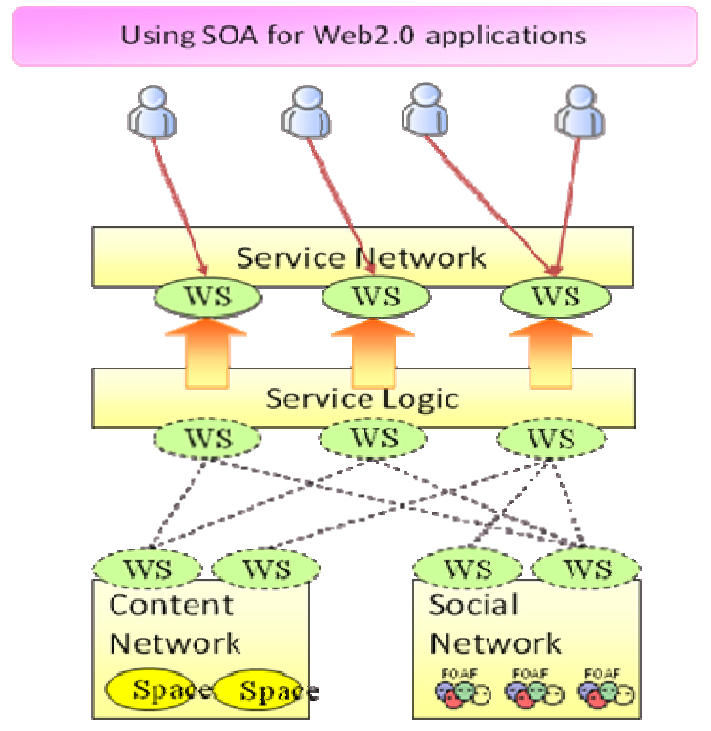

Fig. 2 Using SOA for Web2.0 applications.

All the requests are processed by the Service Logic Layer, which finds the required information and applications for requests. The Content Network is a network consisting of databases and communication support among databases. The Social Network contains the relations for social communities. The following procedure illustrates how it works:

(1)Users send requests to a Service Network;

(2)The Service Network forwards requests to Service Logic Layer via Web service functions. In this step, requests are transferred to objects that can communicate with the Service Logic layer;

(3)When users send requests, Service Network has the ability to generate the desired WS objects according to the requests forwarded by the Service Network;

(4)Service Logic Layer performs the required functions for the requests. It accesses data and information from Content Network using Web service functions. A User can also contact other users using WS objects under the Service Logic Layer;

(5)After the Service Logic Layer has the results for the requests, it sends the results back to the Service Network using Web service functions;

(6)Service Network then sends the results back to the users who sent initial requests.
There are several advantages associated with the design structure:

(1)With this system structure, users can join the desired networks anywhere once they connect to the Internet;

(2)With the characteristics of Web 2.0 with SOA model, users can join the desired networks they need to share or find resources they need easily;

(3)Using WS objects for communication makes it easy for service reusability and service innovation. Users do not need to construct special systems or programs for data accessing and analysis;

(4)Different Service Networks communicate with each other to find and share available resources.

\section{Implementation and Simulation}

Based on the structure of SOA model with Web 2.0 application, the system can be built in a three-tier structure. The lowest level is the sub-networks including Content Network, Social Network, which provides resources for data sharing. The middle level is the Service Logic layer, which provides WS objects. The top level is the Service Network layer that accepts requests or sends request results back to the users.

I use Ajax, Java and other tools to construct the system initially. The first step is to build the system structure with service network, service logic constructed. I also construct the WS object format which is the main object in this system. The functions for applying to the WS objects are needed to be built to apply to the WS objects according to their types. Content network and Social network are for different purposes for data storage management and social media communications. They have the ability to store data and collect data, which then provide information to uses for study purposes.

I then construct a virtual community and work on a simulation for data generation and analysis. Ten thousand nodes with 1000 Web services are used in the simulation. The simulation results present the performance comparison of data sharing and load 
transferring between the structure with the SOA model and the system without it.

\section{Conclusion}

The literature has shown that it is hard to find the required network resources in the Internet for load balance and load service purposes. I propose a new structure for hosts in the computer networks to find resources for web services. This new structure provides a new approach to find resources easily for web services. Especially, when a user needs services which are not very commonly provided in the Internet, with the help of Web 2.0 and SOA, users can find what they need because of the "long tail" property of Web 2.0 and the platform free property of SOA. With this system, uses can find and use the services easily since all the requests and services are constructed with the same web service structure. This can also improves the system performance for the network systems.

This system performance will be evaluated by using a simulation. Usually it is very hard to evaluate the performance for Web 2.0; therefore this project should have great helps in finding the performances for the usage of Web 2.0 and SOA.

\section{References}

[1] Guttman, E., Perkins, C., Veizades, J., and Day, M. 1998. “Service Location Protocol.” Version 2, IEFT, RFC 2165.

[2] Waldo, J. 1999. "The Jini Architecture for Network-Centric Computing." Communication of the ACM 42 (July): 76-82.

[3] Maab, H., "Location-Aware Mobile Application Based on Directory Services.” MOВICOM 97: 23-33.

[4] Yeong, W., Howes, T., and Kille, S. 1995. "Lightweight Directory Access Protocol.” RFC 1777.

[5] Kim, A. J. 2000. Community Building on the Web: Secret Strategies for Successful Online Communities. London: Addison Wesley (ISBN 0-201-87484-9).

[6] Anderson, R. J. 1996. “The Eternity Service.” In Proceedings of the Pragocrypt, 242-52.

[7] Theotokis, S. A., and Spinellis, D. 2004. "A Survey of Peer-to-Peer Content Distribution Technologies.” ACM Computing Sureys $36 \quad$ (4): 335-71. doi:10.1145/1041680.1041681.

[8] Balzer, Y. 2004. "Improve Your SOA Project Plans.” IBM.

[9] Peter, B., England, P., Peinado, M., and Willman, B. 2002. "The Darknet and the Future of Content Distribution.” Presented at the 2002 ACM Workshop on Digital Rights Management.

[10] Bloomberg, J. "Mashups and SOBAs: Which is the Tail and Which is the Dog?” Zapthink.

[11] Holley, C., and Tuggle, E. 2003. "Migrating to a Service-Oriented Architecture.” IBM Developer Works.

[12] Erl, T., "About the Principles." Serviceorientation.org, 2005-2006. 